\title{
Significance of Pseudo-Conductive Hearing Loss and Positional Nystagmus for Perilymphatic Fistula: Are They Related to Third-Window Effects?
}

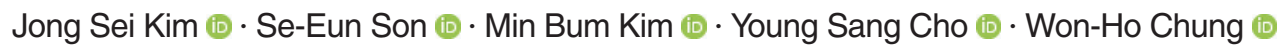 \\ Department of Otorhinolaryngology-Head and Neck Surgery, Samsung Medical Center, \\ Sungkyunkwan University School of Medicine, Seoul, Korea
}

Objectives. Patients' clinical presentation is critical for identifying suspected perilymphatic fistula (PLF). The involvement of third-window lesions in the pathomechanism of PLF has been hypothesized. This study investigated the clinical features of PLF and the relationship of the third-window effect with PLF.

Methods. Sixty patients underwent surgical exploration for suspected PLF and the oval and round windows were reinforced. Clinical features including demographics, pure-tone audiometry (PTA), and videonystagmography were evaluated preoperatively and 1 month postoperatively. Surgical outcomes were analyzed according to the improvement of hearing and vestibular symptoms and signs. The conductive components of PTA (air-bone gap [ABG]) were measured, and the relationship between ABG closure after surgery and hearing improvement was analyzed. In addition, postoperative subjective dizziness was assessed by clinical interviews. Changes in positional nystagmus were analyzed according to $\mathrm{ABG}$ closure and hearing improvement.

Results. ABG at lower frequencies (LFABG; $250 \mathrm{~Hz}, 500 \mathrm{~Hz}, 1,000 \mathrm{~Hz}$ ) was present in 27 patients (45\%). Postoperatively, PTA significantly improved after surgical repair. Among the patients with preoperative LFABG $(n=27), 15(55.5 \%)$ showed postoperative ABG closure and significant improvement in PTA at all frequencies compared with the patients without ABG closure $(P=0.012)$. Subjective dizziness improved in 57 patients $(93.3 \%)$. Positional nystagmus was found in 45 of 49 patients. Multiple canal involvement was more common than single canal involvement $67 \%$ vs. $33 \%$ ). The horizontal semicircular canal was most commonly involved, followed by the posterior and anterior canals. Postoperatively, positional nystagmus disappeared, or the number of involved canals decreased in 22 of 34 patients $(64.7 \%)$.

Conclusion. Pseudo-conductive hearing loss at lower frequencies and positional nystagmus originating from multiple semicircular canals were common findings in PLF. Surgical reinforcement of the oval and round windows improved the hearing threshold accompanied by closure of ABG. A third-window lesion might explain these clinical features of PLF.

Keywords. Perilymph; Nystagmus; Hearing Loss

\section{INTRODUCTION}

- Received September 17, 2020

Revised January 14, 2021

Accepted February 18, 2021

- Corresponding author: Won-Ho Chung

Department of Otorhinolaryngology-Head and Neck Surgery, Samsung

Medical Center, Sungkyunkwan University School of Medicine,

81 Irwon-ro, Gangnam-gu, Seoul 06351, Korea

Tel: +82-2-3410-3571, Fax: +82-2-3410-3879

E-mail: whchung@skku.edu
Perilymphatic fistula (PLF) is an abnormal communication between the middle ear and perilymphatic space in the inner ear; it is commonly caused by internal and external barotrauma such as cough, nose-blowing, or minor head trauma [1]. The main symptoms of PLF are sensorineural hearing loss and vestibular symptoms [2]. Vestibular symptoms such as disequilibrium, motion intolerance, and positional dizziness are most commonly

Copyright () 2021 by Korean Society of Otorhinolaryngology-Head and Neck Surgery.

This is an open-access article distributed under the terms of the Creative Commons Attribution Non-Commercial License (https://creativecommons.org/licenses/by-nc/4.0)

which permits unrestricted non-commercial use, distribution, and reproduction in any medium, provided the original work is properly cited. 
encountered in PLF [3-5].

Due to the lack of objective diagnostic tools, clinical suspicion is important to diagnose PLF. PLF is usually suspected based on clinical features and is confirmed by surgical exploration [2]. Previously, the authors proposed diagnostic criteria for barotraumatic PLF based on clinical manifestations [4]: sudden sensorineural hearing loss following the barotraumatic event and direction-changing positional nystagmus, possibly involving multiple semicircular canals. However, the mechanism of direction-changing positional nystagmus involving multiple semicircular canals has not been well explained, nor has the pathophysiological mechanism of PLF-related symptoms been clearly explained.

Recently, third-window abnormalities were proposed to be involved in several diseases such as superior canal dehiscence, enlarged vestibular aqueduct, abnormal bony dehiscence, and perilymph fistula [6,7].The symptoms consist of sound- or pressureinduced vertigo and/or pseudo-conductive hearing loss at the lower frequencies. In the normal inner ear, both the oval window (OW) and round window (RW) serve as pressure transmitters between the middle ear and perilymphatic spaces. If there is an abnormal bony dehiscence in the inner ear, sound energy from the OW is shunted away through this bony dehiscence, called the third window, thereby decreasing the energy sent to the RW. This aggravates the hearing threshold in air conduction and improves the bone conduction, resulting in pseudo-conductive hearing loss [8]. Wackym et al. [9] reported otic capsule dehiscence syndrome (OCDS), in which the same symptoms as superior canal dehiscence syndrome (SCDS) occurred in patients with no otic capsule dehiscence on imaging. They described patients with OCDS as having similar symptoms to those with SCDS, such as dizziness, auditory symptoms, headache, and cognitive dysfunction. Surgical reinforcement of the RW was proposed as a way to effectively improve the symptoms not by stopping the perilymph leak, but by closing the third window to change the biomechanical properties of the perilymphatic fluids. They also suggested the cochlea modiolus as a possible location to create a third window [9].

In our series of patients with PLF, sensorineural hearing loss and dizziness were the most common symptoms; hearing loss

\section{H I G H L I G H T S}

- Positional nystagmus disappeared after surgical reinforcement of the round and oval windows.

- After surgical reinforcement, subjective dizziness improved and the number of the involved canals decreased.

- Patients who showed postoperative air-bone gap (ABG) closure presented a significant improvement in pure-tone audiometry at all frequencies compared with patients without ABG closure. was improved by early surgical repair. In contrast, regarding vestibular symptoms, the patients showed direction-changing positional nystagmus involving multiple semicircular canals $[4,5,10]$. The vestibular symptoms recovered with surgical reinforcement of the RW and OW [3,11]. Interestingly, although hearing recovery was related to surgical timing, improvement of vestibular symptoms was not [3].

Neurotologic findings related to positional nystagmus in patients with PLF have rarely been described in detail. To detect PLF, pressure-induced vertigo and nystagmus (Hennebert's sign) and sound-induced vertigo and nystagmus (the Tullio phenomenon) have been used $[12,13]$. In addition, dynamic posturography was used to quantify the postural disturbance induced by sound [14] or pressure [15] changes in the inner ear. However, positional nystagmus as a possible cause of disabling dizziness in PLF has not been described.

In this study, we hypothesized that a third-window lesion might be involved in the pathomechanism of PLF. If patients have underlying otic capsule dehiscence without radiologically proven defects, barotraumatic events would cause the symptoms and signs of third-window abnormalities. These abnormalities are mainly related to the auditory symptoms of bone conduction hypersensitivity (pseudo-conductive hearing loss). In addition, positional nystagmus might be related to third-window abnormalities. The purpose of this study was to investigate the clinical features related to PLF, placing a special emphasis on pseudo-conductive hearing loss and positional nystagmus that might be associated with third-window effects.

\section{MATERIALS AND METHODS}

The Institutional Review Board of Samsung Medical Center approved this study (IRB No. 2015-12-101). This study was exempts from requirements for informed consent due to the retrospective, observational nature.

A total of 60 patients (62 ears) was retrospectively reviewed. The patients all underwent surgical exploration on suspicion of PLF between January 2005 and December 2019. The diagnostic criteria previously proposed were used to indicate barotraumatic PLF [4]. The diagnostic criteria of barotraumatic PLF include preceding history of barotrauma, sudden auditory symptoms (sensorineural hearing loss, tinnitus, and/or ear fullness), and one or more features of dizziness accompanying with auditory symptoms. The features of dizziness consist of time difference between the onset of auditory symptoms and dizziness, dizziness aggravated by positional change, and positional nystagmus. The characteristics of positional nystagmus is multi-directional, small amplitude, long duration, no reversibility, and no response to otolith repositioning maneuver. In addition, we included 11 patients with idiopathic causes who showed the same clinical features. 
The etiology of trauma was categorized into two types, internal and external. Internal trauma can be further subdivided into two subtypes, implosive (nose blowing, flying, diving) and explosive (Valsalva, heavy lifting) [1]. External trauma included minor head trauma such as slap injury. Pure tone audiogram and videonystagmography (VNG) were assessed preoperatively and 1 month after surgical repair. For hearing assessment, a pure tone average of four frequencies $(0.5 \mathrm{k}, 1 \mathrm{k}, 2 \mathrm{k}$, and $4 \mathrm{kHz})$ was used. In addition, air-bone gap at lower frequencies (LFABG; 250, 500, and $1 \mathrm{kHz}$ ) was measured. The significant LFABG was defined to be greater than average $15 \mathrm{~dB}$ at 250,500 , and $1 \mathrm{kHz}$. The definition of LFABG has been reported in some of the Meniere disease research [16,17], but it was the first time covered in PLF research. VNG was tested for identifying the spontaneous and positional nystagmus. The positional tests including Dix-Hallpike and supine head roll test were performed and the nystagmus was recorded using VNG. Preoperative caloric tests were performed in 37 patients using an infrared video-oculographic system (Micromedical Technologies, Chatham, IL, USA) and a BrooklerGrams closed-loop irrigation unit (Grams Medical, Costa Mesa, CA, USA). Canal paresis (CP) equal to or greater than $26 \%$ was considered abnormal. Temporal bone computed tomography was performed in every patient. Patients with inner ear anomaly or middle ear disease were excluded from this study.

In every patient, surgical exploration was conducted via an endaural approach under local anesthesia. After tympanomeatal flap elevation, both the RW and OW were carefully examined for evidence of PLF such as perilymph leak or pooling, membrane tearing, and fibrous mesh formation. When appropriate, an endoscope was used for better visualization. To induce perilymph leak or dizzy symptoms, the Valsalva maneuver or stapes palpation was applied. After inspecting the evidence of PLF, both the RW and OW were covered and reinforced with soft tissue, cartilage pieces, and fibrin glue regardless of fistula presence.

After surgical procedures, patients were categorized into definite and probable PLF categories according to our criteria [4]. Definite PLF was defined when any evidence of perilymph fistula was found during exploration. For probable PLF, no definitive evidence of PLF was found.

Treatment outcomes were analyzed based on hearing improvements, changes of subjective dizziness, and nystagmus. Hearing improvements were defined as a postoperative four-frequency pure-tone average $(0.5 \mathrm{k}, 1 \mathrm{k}, 2 \mathrm{k}$, and $4 \mathrm{kHz})$ for bone conduction less than or equal to $25 \mathrm{~dB}$, or a hearing gain greater than or equal to $15 \mathrm{~dB}$. According to the significant presence of LFABG, patients were divided into two groups: presence and absence of LFABG. In addition, the group of positive LFABG was subdivided into two groups with or without postoperative LFABG closure. LFABG closure was defined as LFABG less than or equal to $5 \mathrm{~dB}$. The two groups were compared according to hearing improvement.

The degree of subjective dizziness was assessed by question- ing the patients in the outpatient clinic. Improvements in subjective dizziness were divided into complete, partial, and no improvement. Complete improvement was defined as no dizzy spell at all after surgery. Partial improvement was defined as a symptom that was still present but was better than before operation. No improvement was defined as no change at all after surgery.

The involved canal was analyzed by the direction of nystagmus using the positional tests. According to Ewald's first law, the direction of nystagmus followed the stimulated semicircular canal. Stimulation of the horizontal canal induced horizontal nystagmus; stimulation of the posterior and anterior canals induced up-beating torsional and down-beating torsional nystagmus, respectively. To assess the change in nystagmus pattern, involved canals were compared preoperatively and at 1 month after surgery. Additionally, the pattern of positional nystagmus was compared according to the closure of LFABG and hearing improvement.

Statistical analysis was performed using IBM SPSS ver. 26.0 (IBM Corp., Armonk, NY, USA). Wilcoxon signed-rank test for nonparametric data was employed for analysis of hearing outcomes. The Mann-Whitney test for nonparametric data was employed for group analysis between two groups according to positive or negative ABG and its improvement. The Fisher's exact test was employed for analysis of the difference in recovery rate of vestibular symptom between definitive and probable PLF. The presence of positional nystagmus and multiple canal involvement were compared before and after surgery by using the McNemar test. A $P$ value $<0.05$ was considered statistically significant.

\section{RESULTS}

\section{Patient demographics}

The patients' demographic information is described in Table 1 . Sixty patients (34 men and 26 women) from 12 to 80 years old (mean, $44.0 \pm 20.2$ years) were enrolled. Internal trauma ( $\mathrm{n}=38$, $63.3 \%$ ) was the most common cause of PLF, followed by external trauma ( $n=11,18.3 \%)$ and idiopathic causes $(n=11,18.3 \%)$. There were 28 cases $(46.6 \%)$ of definite PLF, in which the evidence of PLF was confirmed intraoperatively. In definite PLF, the RW (15 cases) was a more common fistula site than the OW (8 cases). In five patients $(17.8 \%)$, it was not clearly identified whether the OW or RW was the leakage site. Thirty-two (53.3\%) patients in whom PLF was not evident were classified as having probable PLF. The median interval from symptom onset to surgical repair was 9.0 days (interquartile range, 4-20 days).

\section{Clinical features Hearing loss}

Every patient had sudden onset of auditory symptoms such as hearing loss, ear fullness, or tinnitus. Preoperatively, the average 
Table 1. Demographic information of 60 patients who underwent surgical repair under suspicion of PLF

\begin{tabular}{|c|c|}
\hline Demographic category & Value $(n=60)$ \\
\hline \multicolumn{2}{|l|}{ Sex } \\
\hline Male & $34(56.6)$ \\
\hline Female & $26(43.3)$ \\
\hline Age (yr) & $44.0 \pm 20.2(12-80)$ \\
\hline \multicolumn{2}{|l|}{ Lesion side } \\
\hline Right & $31(51.6)$ \\
\hline Left & $29(48.3)$ \\
\hline Surgical timing after symptom onset (day) & $9(0-671)$ \\
\hline \multicolumn{2}{|l|}{ Etiologic event } \\
\hline Internal & $38(63.3)$ \\
\hline External & $11(18.3)$ \\
\hline Idiopathic & $11(18.3)$ \\
\hline \multicolumn{2}{|l|}{ Classification } \\
\hline Definite & $28(46.6)$ \\
\hline Probable & $32(53.3)$ \\
\hline \multicolumn{2}{|l|}{ Fistula site in definite PLF $(n=28)$} \\
\hline OW & $8(28.5)$ \\
\hline RW & $15(53.5)$ \\
\hline Unidentified & $5(17.8)$ \\
\hline \multicolumn{2}{|l|}{ Pure-tone average (dB) } \\
\hline Preoperative & $81.8 \pm 23.7(24-123)$ \\
\hline Postoperative & $63.4 \pm 30.7(13-117)$ \\
\hline \multicolumn{2}{|l|}{ Hearing improvement $(n=57)$} \\
\hline Yes $(\geq 15 \mathrm{~dB}$ in PTA) & $33(57.8)$ \\
\hline No $(<15 \mathrm{~dB}$ in PTA $)$ & $24(42.1)$ \\
\hline \multicolumn{2}{|l|}{ Presence of LFABG } \\
\hline Yes & $27(45.0)$ \\
\hline No & $33(55.0)$ \\
\hline \multicolumn{2}{|l|}{ Postoperative LFABG closure $(n=27)$} \\
\hline Yes & $15(55.5)$ \\
\hline No & $12(44.4)$ \\
\hline \multicolumn{2}{|l|}{ Caloric response $(n=37)$} \\
\hline Normal $(\mathrm{CP}<25)$ & $21(56.7)$ \\
\hline Decreased $(\mathrm{CP} \geq 25)$ & $16(43.2)$ \\
\hline \multicolumn{2}{|l|}{ Spontaneous nystagmus, preoperative $(n=60)$} \\
\hline Yes & $21(35.0)$ \\
\hline No & $39(65.0)$ \\
\hline \multicolumn{2}{|l|}{ Positional nystagmus, preoperative $(n=49)$} \\
\hline Yes & $45(91.8)$ \\
\hline No & $4(8.1)$ \\
\hline \multicolumn{2}{|l|}{ Positional nystagmus, preoperative $(n=45)$} \\
\hline Lateral & $37(82.2)$ \\
\hline Anterior & $15(33.3)$ \\
\hline Posterior & $25(55.5)$ \\
\hline \multicolumn{2}{|l|}{ Positional nystagmus, postoperative $(n=34)$} \\
\hline Lateral & $16(47.0)$ \\
\hline Anterior & $7(20.5)$ \\
\hline Posterior & $8(23.5)$ \\
\hline
\end{tabular}

Values are presented as number (\%), mean \pm standard deviation (interquartile range), or mean (range).

PLF, perilymphatic fistula; OW, oval window; RW, round window; PTA, pure-tone audiometry; LFAGB, air-bone gap at lower frequencies, CP, canal paresis.

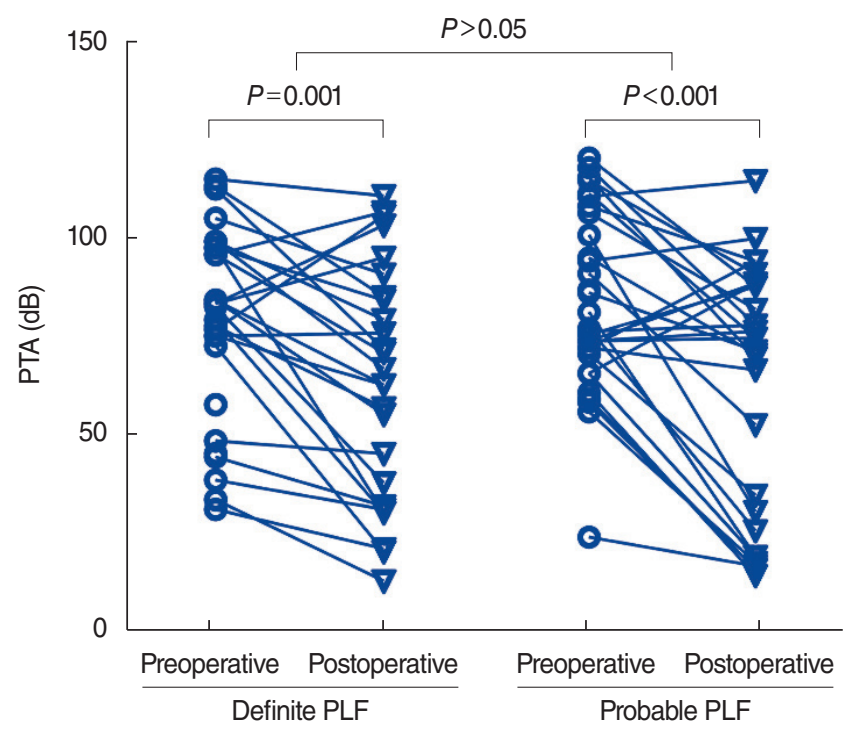

Fig. 1. Postoperative hearing outcomes at 1 month after surgical repair in definite and probable perilymphatic fistula (PLF). In both groups, the pure-tone audiometry (PTA) significantly improved. However, there was no significant between-group difference.

pure-tone audiometry PTA was $81.8 \pm 23.7 \mathrm{~dB}$ (range, 24-123 dB). The configuration of the audiogram was downsloping, with greater hearing loss at higher frequencies. The PTA was not significantly different according to the etiology of trauma, fistula site, or between definite and probable PLF (data not shown).

Twenty-seven patients (45\%) showed preoperative LFABG. The groups with and without LFABG showed no significant differences in clinical features such as PTA, the etiology of trauma, surgical timing, and fistula site. Postoperatively, 33 of 57 patients $(57.8 \%)$ showed hearing gain at 1 month after surgery (three patients who had no postoperative audiometry were excluded). Postoperatively, the average PTA was $63.3 \pm 30.7 \mathrm{~dB}$ (range, 13$117 \mathrm{~dB}$ ). PTA significantly improved after surgical repair in both definite and probable PLF cases (Fig. 1). The improvement of hearing after surgical repair was significantly related to surgical timing, but not to other factors such as etiology of trauma or the fistula site (data not shown).

Among the patients $(n=27)$ with preoperative LFABG, 15 $(55.5 \%)$ showed postoperative closure of the LFABG. The patients with postoperative LFABG closure showed significant improvements in PTA at all frequencies $(P=0.012)$. In contrast, PTA did not significantly improve in patients who did not have LFABG closure. Postoperatively, the mean hearing thresholds for the two groups were $52.5 \pm 29.2 \mathrm{~dB}$ (range, 18-102 dB) for the LFABG closure group and $81.6 \pm 18.4 \mathrm{~dB}$ (range, $45-107 \mathrm{~dB}$ ) for the LFABG non-closure group (Fig. 2). In Fig. 3, two audiograms are presented to demonstrate the preoperative LFABG and hearing improvement with LFABG closure after surgical repair. 

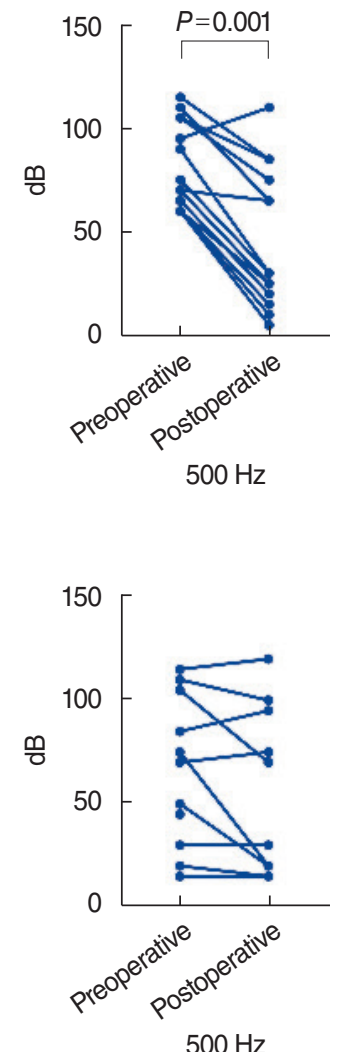

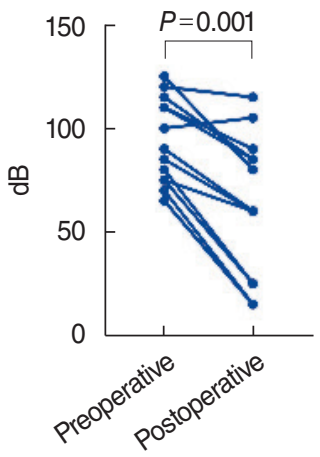

$1 \mathrm{kHz}$

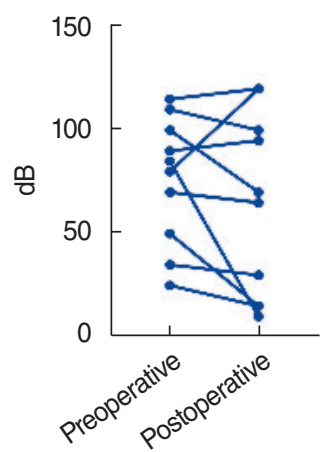

$1 \mathrm{kHz}$

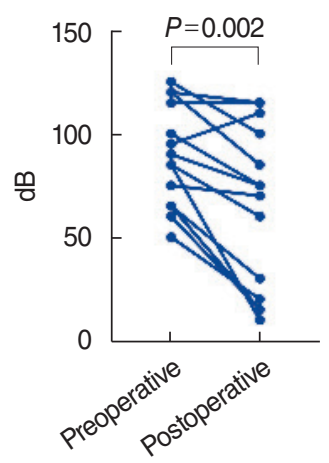

$2 \mathrm{kHz}$

LFABG non-closure

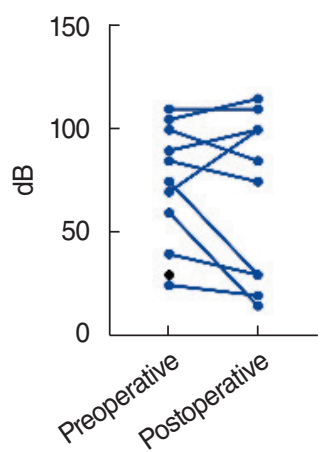

$2 \mathrm{kHz}$

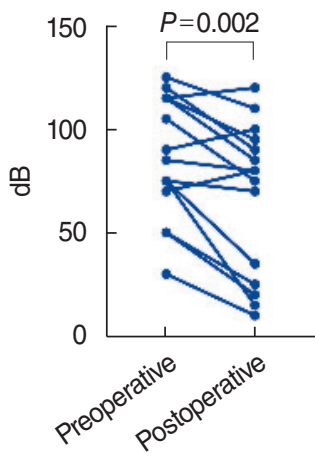

$4 \mathrm{kHz}$

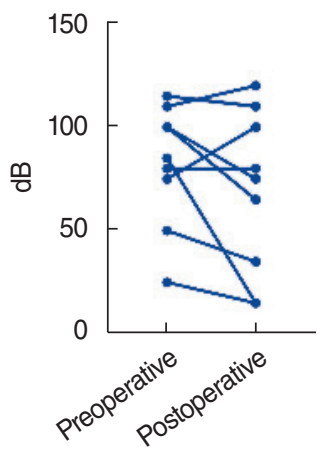

$4 \mathrm{kHz}$

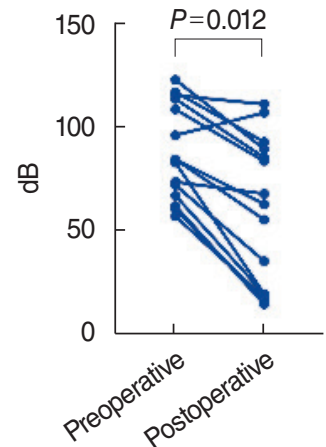

PTA

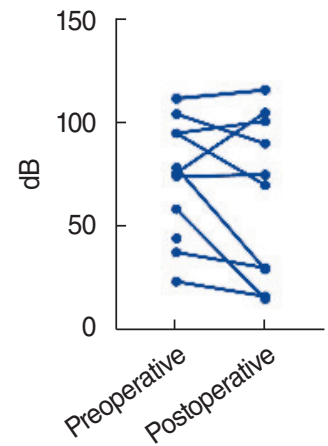

PTA

Fig. 2. Postoperative hearing outcomes at 1 month after surgical repair in the air-bone gap at lower frequencies (LFABG) closure and non-closure groups. (A) In the LFABG closure group, hearing significantly improved at each frequency after surgery. (B) In the LFABG non-closure group, hearing did not improve at each frequency. PTA, pure-tone audiometry.
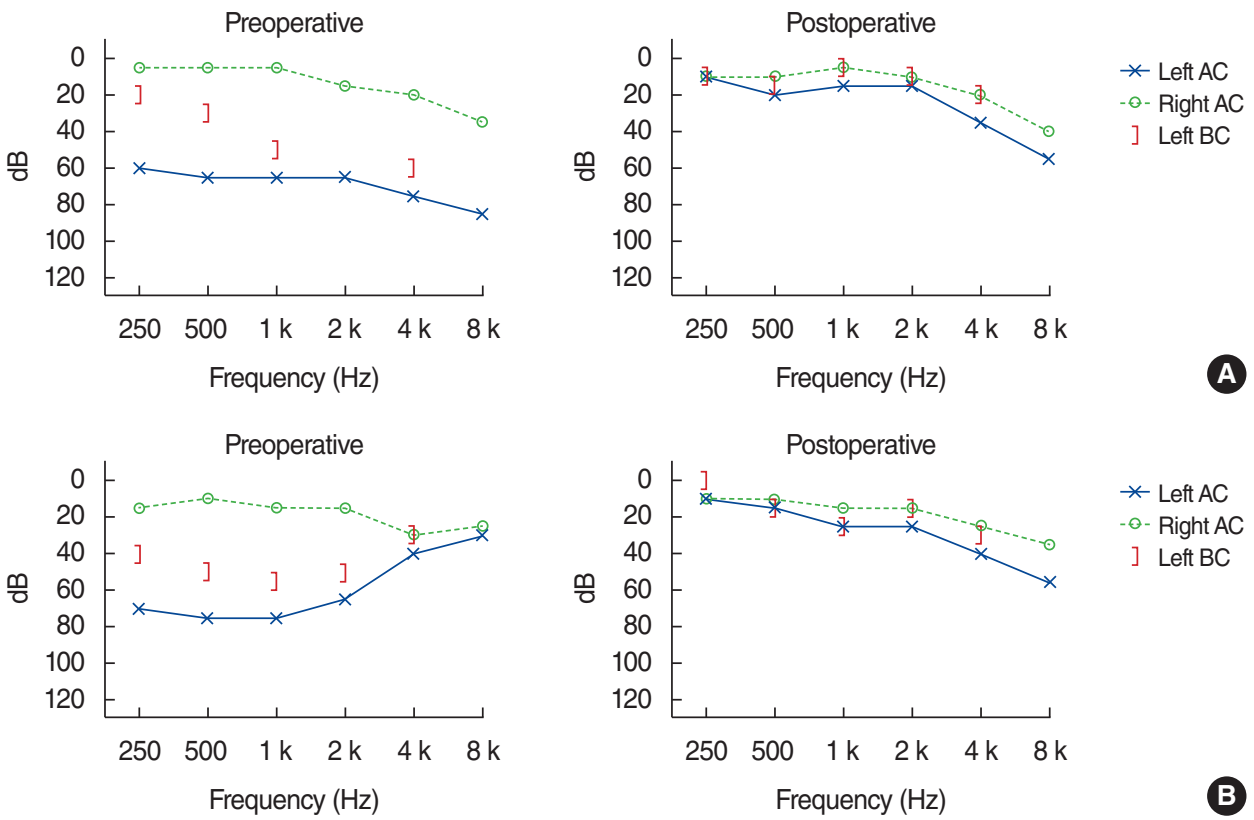

Fig. 3. Examples of preoperative pseudo-conductive hearing loss at lower frequencies and postoperative hearing results in patient $1(A)$ and patient 2 (B). The air-bone gap at lower frequencies (LFABG) was closed with hearing improvement after surgical repair. AC, air conduction; $\mathrm{BC}$, bone conduction. 


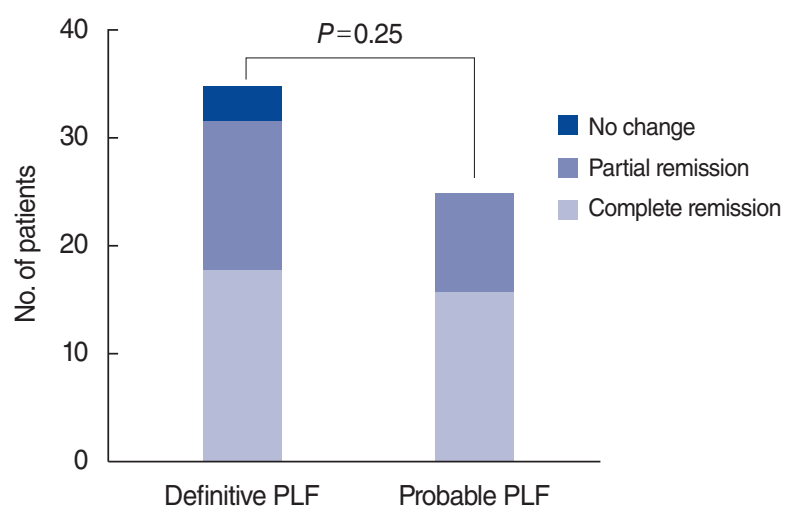

Fig. 4. Changes in subjective dizziness after surgical repair of perilymphatic fistula (PLF). Subjective dizziness improved in most patients. There was no significant difference between definite and probable PLF.

\section{Vestibular symptoms and signs}

Subjective dizziness improved after surgery in most patients. At 1 month postoperatively, subjective dizziness had completely subsided in 34 patients, and partially improved in 23 patients (Fig. 4). Three patients did not improve, two of whom underwent revision surgery because of recurrent dizziness with positional nystagmus. There was no significant difference in the recovery rate between definitive and probable PLF cases $(P=0.25)$.

VNG recordings were performed preoperatively and 1 month postoperatively for 34 patients, and caloric test results were gathered preoperatively for 37 patients. Preoperatively, spontaneous nystagmus was found in 21 of 60 patients (35\%). The direction was toward either the lesion side or the normal side. Some patients showed spontaneous vertical beating nystagmus, which likely originated from the otolithic organs. The velocity of spontaneous nystagmus was not fast enough for the patients to experience severe whirling vertigo. Instead, patients noted postural disturbance and unsteadiness. On caloric tests, 16 patients showed abnormal CP but did not manifest the symptoms and signs of acute spontaneous vestibular loss, such as strong whirling vertigo with spontaneous nystagmus and bedside head impulse test abnormality. In addition, spontaneous nystagmus resolved immediately after surgical repair in every patient.

Preoperatively, positional nystagmus was found in 45 of 49 patients $(91.8 \%)$ who underwent preoperative VNG. In 15 of 45 patients $(33 \%)$, single-directional nystagmus was recorded. Horizontal positional nystagmus (horizontal canal involvement) was most common (10/15), with down-beating torsional (anterior canal involvement) nystagmus found in two cases and up-beating torsional nystagmus found in three cases (posterior canal involvement). In 30 of 45 patients (67\%), multiple-directional nystagmus was recorded, indicating multiple canal involvement. The combination of horizontal and posterior canals was most common ( $n=17)$ followed by the combination of horizontal and anterior canals $(n=8)$. Involvement of three canals was encoun-

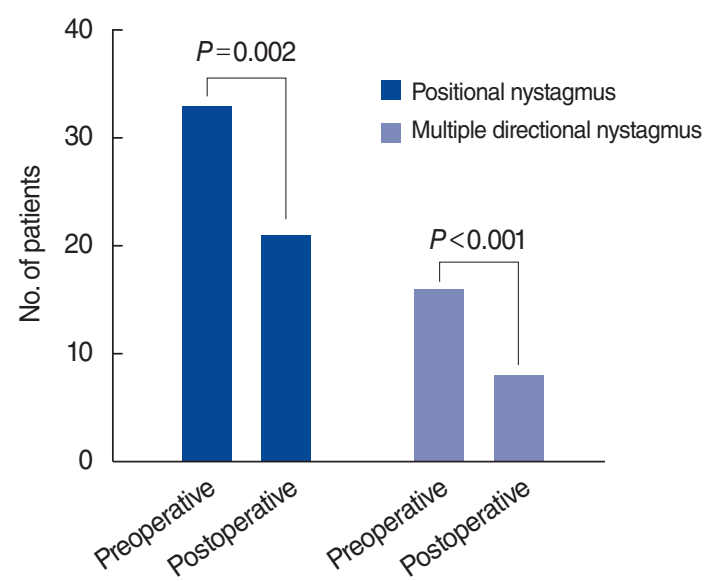

Fig. 5. Changes in positional nystagmus and the incidence of multiple canal involvement after surgical repair of perilymphatic fistula. Positional nystagmus was found in $97.0 \%$ of patients preoperatively, and the number of patients significantly decreased after surgery. In addition, the incidence of multiple-canal involvement significantly decreased after surgery.

tered in two cases. Among 45 cases, 34 patients underwent VNG 1 month postoperatively. The incidence of positional nystagmus and multiple canal involvement significantly decreased after surgery in 22 of 34 patients (64.7\%) (Fig. 5). However, the ratio of involved canals in multiple canal did not change after surgical repair.

\section{DISCUSSION}

In our series of PLF patients $(n=60)$, the main symptoms were sudden auditory symptoms and dizziness. The auditory symptoms included hearing loss, tinnitus, and/or ear fullness. The symptoms usually followed immediately after a barotraumatic event; however, subjective detection of hearing loss can be delayed and hearing loss can be progressive [4]. The severity of hearing loss was diverse, and the audiograms tended to have a downsloping configuration. Hearing loss was significantly improved by early surgical repair [10]. In addition, LFABG was detected in 27 patients $(45 \%)$.

The mechanism of hearing loss in PLF is considered to be sudden intracochlear pressure change by perilymph leak and a secondary lesion to the surrounding membrane labyrinth, such as Reissner's membrane. The degree of hearing loss varies depending on the severity of damage to the membranous labyrinth. In an animal study, artificial PLF induced an intracochlear pressure change without a hearing threshold shift, and the pressure could be restored by spontaneous healing of RW in 4 days [18]. However, if the damage was severe enough to induce secondary damage to the membranous labyrinth, especially to Reissner's membrane, the mixture of perilymph and endolymph caused hair cell 
damage, resulting in permanent hearing loss [19]. Most hearing loss recovered after the fistula site was closed, either spontaneously or by early surgical closure, resulting in secondary healing of the membranous labyrinth [20]. The high-frequency area in the cochlea is more vulnerable because of its proximity to the PLF site of the OW or RW.

The mechanism of LFABG is not clear. Of the 60 total patients, 27 had LFABG. Among them, 15 (55.5\%) showed LFABG closure after surgery; with LFABG closure, the hearing thresholds significantly improved. Therefore, LFABG might reflect pseudoconductive hearing loss, especially at lower frequencies. Pseudoconductive hearing loss is a common symptom in third-window lesions. With third-window effects, the air conduction threshold is elevated by acoustic shunting, and the bone conduction threshold is improved by lowering impedance in the scala vestibuli [8]. There are potential third windows in the inner ear such as the vestibular aqueduct, cochlear aqueduct, and foramina for blood vessels. Goodhill et al. [1] described the role of the cochlear aqueduct and the internal auditory canal as potential pathways from the subarachnoid space to the perilymphatic space. Increased cerebrospinal fluid pressure due to barotraumatic events transmits the pressure through these pathways to damage the inner ear structures including the RW, OW, or labyrinthine membranes and cause barotraumatic PLF. In addition, Kohut et al. [21] reported a potential communication tract between the vestibule and middle ear in the fissula ante fenestram and posterior canal ampulla in the temporal bones of PLF patients. People with these congenital defects may be more vulnerable to PLF caused by barotraumatic events. Wackym et al. [9] reported OCDS without radiographically visible dehiscence, in which the clinical presentation was similar to that of SCDS. They described symptom relief by surgical reinforcement of the RW. In our series, patients who showed postoperative ABG closure had improved hearing compared with patients who did not. Therefore, the conductive components closed with the hearing improvement achieved by RW and OW reinforcement. Without hearing improvement, the conductive components remained open. Therefore, by reducing third-window effects, hearing loss might improve. LFABG may be a key clinical feature that associates the third-window effect with PLF. However, in this study, only 33 patients (55\%) showed LFABG. There are two possible reasons for this result. First, 32 (53.3\%) probable PLF patients did not present the typical symptoms of PLF, especially in LFABG. Second, it is possible that LFABG disappeared in patients who had symptoms occurring after surgery for a longer period of time. Patients remember the onset of symptoms clearly, but often cannot remember when they experienced external trauma. In this study, since idiopathic PLF patients were included, it was difficult to clarify the interval between trauma onset and neurotologic tests. However, if we assume that symptoms started immediately after the occurrence of trauma and that surgical reinforcement was performed within 1 or 2 days after the tests, the interval time between symptom onset and surgical repair would present a similar duration to the interval time between the trauma and tests. Although not presented in the results of the current study, the difference in the interval time according to the presence of LFABG was not statistically significant $(P=0.97)$. We therefore suggest that further research on the clinical significance of LFABG is required.

To summarize our results on hearing outcomes in PLF, the degree of hearing loss varied depending on the etiology of barotraumatic events. Early surgical repair resulted in the improvement of hearing loss. The presence of pseudo-conductive hearing loss at lower frequencies may indicate a possible role of the third-window mechanism in the pathomechanism of PLF. Individual susceptibility differed according to the potential pathways to be able to function as the third window. RW reinforcement may improve hearing loss either by stopping the continuous perilymph leak or by reducing the third-window effects.

Vestibular symptoms in PLF patients are common disabling symptoms $[2,7,22]$. Vertigo can be induced by sound (the Tullio phenomenon) or pressure changes (Hennebert's sign) $[7,8]$. However, in our series, Hennebert's sign and the Tullio phenomenon were very rare (data not shown). The symptoms were usually postural disturbance and positional dizziness, which were not explained by endolymphatic hydrops or by vestibular hypofunction. The vestibular symptoms usually occurred immediately after the barotraumatic events and continued for a long period of time. The vestibular symptoms improved shortly after surgical repair of PLF regardless of surgical timing.

In this study, we included 11 idiopathic cases suspected to have PLF. These patients suffered from the same clinical features of barotraumatic PLF patients-sudden and progressive sensorineural hearing loss with multiple-directional positional nystagmus. During exploration, we found definite evidence of perilymph leak in six of 11 patients (54.5\%). Complete and partial improvement of vestibular symptoms was found in three (27.2\%) and eight $(72.7 \%)$ of these patients, respectively. The presymptomatic events included head rotation and breast feeding. To be clear, this can act as a confounding factor in analyzing the results. Idiopathic PLF is also called spontaneous PLF. Some studies have reported the possibility of developing PLF without an obvious trauma history $[23,24]$. This result does not mean that idiopathic PLF could occur without trauma, but it may be that the trauma is too insignificant for the patient to recognize. Since the trauma history can be uncertain because it relies entirely on the patient's memory, we decided to include the idiopathic PLF patients in this study. Consequentially, patients who satisfied the clinical features of PLF in the diagnostic criteria but did not satisfy the condition of having a trauma history were included.

As previously reported on preoperative neurotologic testing, spontaneous nystagmus (35\%) and positional nystagmus were the most common findings $[1,22]$. Spontaneous nystagmus had a slow velocity, and the direction was horizontal or vertical. Because spontaneous nystagmus was not related to caloric loss, it 
may have been caused by stimulation of the otolithic organs or semicircular canals resulting from pressure change, rather than unilateral vestibular loss.

The most common physical finding in this study was positional nystagmus, which may be related with concomitant benign paroxysmal positional vertigo (BPPV) [3]. Characteristically, positional nystagmus from trauma is different from positional nystagmus due to BPPV. Both the Dix-Hallpike test and supine head roll test induced positional nystagmus with subjective dizziness. In addition, the direction of positional nystagmus varied. For example, three directions of nystagmus (up-beating torsional, downbeating torsional, and horizontal) were induced simultaneously by different positional tests (Supplementary Video 1). As the direction of nystagmus is followed by canal stimulation according to Ewald's first law, this finding implied that three semicircular canals could be simultaneously stimulated by different positional tests. Postoperatively, after RW and OW reinforcement, patients' symptoms improved, and the intensity of positional nystagmus subsided or decreased.

The mechanism of positional nystagmus can be explained by "the floating labyrinth," as proposed previously [25]. In animal models of PLF, the trabecular mesh surrounding the endolymphatic membrane was disrupted by pressure change in the perilymphatic space. As a result, the endolymphatic membrane collapsed to the crista ampullaris and can be shifted by a positional change. This floating labyrinth could potentially occur in any of the canals or otolithic organs depending on the location and severity of pressure change, which would explain positional nystagmus involving multiple canals. In our study, the horizontal semicircular canal was most frequently involved, followed by the posterior and anterior canals because of their proximity to the RW or OW. Nomura et al. [25] described a reduction in the caloric response by the floating labyrinth and not by actual vestibular loss. However, the floating labyrinth theory alone cannot explain why the symptoms and signs were improved by RW or OW reinforcement.

Wackym et al. [9] described OCDS without radiographically visible dehiscence, in which the clinical presentation was similar to that of SCDS. These patients had a history of head trauma and complained of dizziness, headache, and cognitive dysfunction. The symptoms were relieved by surgical reinforcement of the RW regardless of surgical timing. The vestibular symptoms in third-window lesions were mostly improved by RW reinforcement surgery $[9,26]$.

Regarding vestibular symptoms, a third-window lesion on one side shunts acoustic energy and causes an asymmetric displacement of fluid, resulting in larger deflections of vestibular sensors by sound or pressure or even positional change [5]. In SCDS, which is a typical example of third-window effects, nystagmus was induced by sound or pressure or vibration. Nystagmus is caused by stimulation of not only the superior canal, but also the posterior canal and/or horizontal canal. Therefore, up-beat- ing, down-beating, and horizontal nystagmus was documented, depending on the location of dehiscence [27]. The same mechanism might be applied to the PLF by positional change. If there is a continuous perilymph leak to the middle ear, positional change toward the lesion side would induce larger fluid movement, resulting in positional nystagmus. This can occur in any of the canals, thus possibly affecting any crista of the three semicircular canals. In addition, even though the PLF healed spontaneously, the stiffness change of the RW with third-window lesions would cause the same positional nystagmus in any canal. Therefore, by reinforcing the RW, the third-window effects would be reduced, and symptoms would subside.

In our series, the preoperative nystagmus pattern suggested that multiple canals were stimulated by positional changes, and that nystagmus subsided due to RW or OW reinforcement. Two patients underwent revision surgery due to recurrent vestibular symptoms and positional nystagmus several months after the first surgery. In revision surgery, the soft tissue previously packed for RW or OW repair was no longer observed. After repacking the RW and OW, the patients' symptoms improved. Therefore, the possible explanations for vestibular symptoms in relation to the third-window effects are (1) long-standing postural instability with multidirectional positional nystagmus without acute vestibular loss or (2) relief of the symptoms and signs by surgical reinforcement of the RW or OW.

There are limitations of this study. Despite our explanations and data, direct evidence of the third-window effect is still lacking because the demographic characteristics of the patients were diverse regarding the etiology of trauma, location of trauma, severity of pressure change, duration of symptoms, and patient susceptibility. Due to the limitations of the retrospective design, we could not analyze subjective indicators of severity, disability, and functional limitation due to dizziness. Instead, we only evaluated the subjective complaints of patients with simple questions about the status of dizziness in the outpatient clinic. Subjective indicators of dizziness, such as the Dizziness Handicap Inventory, Activities-Specific Balance Confidence Scale, the Functional Level scale of the American Academy of Otolaryngology-Head and Neck Surgery, and the Vestibular Disorders Activities of Daily Living Scale, could be utilized to evaluate patients' subjective dizziness. These modalities could be used in further research. In addition, our data were not sufficient to explain the mechanism of third-window lesions. The number of patients was also small, other electrophysiological data such as vestibular evoked myogenic potential (VEMP) were lacking, and the follow-up period was short. Electrophysiological data such as VEMP would be helpful, but those data were unfortunately not available in our series. Regarding cervical VEMP (cVEMP), lowering of the threshold in SCDS is a well-documented finding supporting a third-window lesion, but in PLF, only a few papers have reportedVEMP results $[28,29]$. They showed lowered cVEMP thresholds in four cases of PLF. More studies are needed to con- 
firm this. However, we did demonstrate that pseudo-conductive hearing loss and multiple directional positional nystagmus without vestibular loss related to PLF might be partly related to a third-window lesion. This relationship has not been reported before. Stiffness change of the RW or OW associated with possible temporal bone dehiscence unrecognized by temporal bone computed tomography might explain the PLF symptoms associated with third-window lesions.

\section{CONFLICT OF INTEREST}

No potential conflict of interest relevant to this article was reported.

\section{ACKNOWLEDGMENTS}

The authors are thankful to all staff members, doctors, and statistical consultants who were involved in this study.

\section{ORCID}

Jong Sei Kim

https://orcid.org/0000-0002-1798-817X

Se-Eun Son

https://orcid.org/0000-0002-5104-0462

Min Bum Kim

https://orcid.org/0000-0001-5418-5176

Young Sang Cho

https://orcid.org/0000-0002-4040-7206

Won-Ho Chung

\section{AUTHOR CONTRIBUTIONS}

Conceptualization: WHC. Data curation: SES. Formal analysis: JSK. Methodology: JSK, MBK, YSC. Project administration: all authors. Visualization: JSK. Writing-original draft: JSK. Writingreview \& editing: all authors.

\section{SUPPLEMENTARY MATERIALS}

Supplementary materials can be found via https://doi.org/10. 21053/ceo.2020.01942.

\section{REFERENCES}

1. Goodhill V, Brockman SJ, Harris I, Hantz O. Sudden deafness and labyrinthine window ruptures: audio-vestibular observations. Ann Otol Rhinol Laryngol. 1973 Jan-Feb;82(1):2-12.

2. Hornibrook J. Perilymph fistula: fifty years of controversy. ISRN Otolaryngol. 2012 Jul;2012:281248.

3. Hornibrook J.A balance test for chronic perilymph fistula. Int J Oto-

laryngol. 2012;2012:163691.

4. Choi JE, Moon IJ, Kim H, Lee K, Cho YS, Chung WH. Diagnostic criteria of barotraumatic perilymph fistula based on clinical manifestations. Acta Otolaryngol. 2017 Jan;137(1):16-22.

5. Ahn J, Son SE, Choi JE, Cho YS, Chung WH. Surgical outcomes on hearing and vestibular symptoms in barotraumatic perilymphatic fistula. Otol Neurotol. 2019 Apr;40(4):e356-63.

6. Ho ML, Moonis G, Halpin CF, Curtin HD. Spectrum of third window abnormalities: semicircular canal dehiscence and beyond. AJNR Am J Neuroradiol. 2017 Jan;38(1):2-9.

7. Ward BK, Carey JP, Minor LB. Superior canal dehiscence syndrome: lessons from the first 20 years. Front Neurol. 2017 Apr;8:177.

8. Merchant SN, Rosowski JJ. Conductive hearing loss caused by thirdwindow lesions of the inner ear. Otol Neurotol. 2008 Apr;29(3):282-9.

9. Wackym PA,Wood SJ, Siker DA, Carter DM. Otic capsule dehiscence syndrome: superior semicircular canal dehiscence syndrome with no radiographically visible dehiscence. Ear Nose Throat J. 2015 Aug; 94(8):E8-24.

10. Park GY, Byun H, Moon IJ, Hong SH, Cho YS, Chung WH. Effects of early surgical exploration in suspected barotraumatic perilymph fistulas. Clin Exp Otorhinolaryngol. 2012 Jun;5(2):74-80.

11. Alzahrani M, Fadous R, Dufour JJ, Saliba I. Perilymphatic fistulas: can we predict the diagnosis? Eur Arch Otorhinolaryngol. 2015 Aug; 272(8):1885-91.

12. Black FO, Lilly DJ, Nashner LM, Peterka RJ, Pesznecker SC. Quantitative diagnostic test for perilymph fistulas. Otolaryngol Head Neck Surg. 1987 Feb;96(2):125-34.

13. Daspit CP, Churchill D, Linthicum FH Jr. Diagnosis of perilymph fistula using ENG and impedance. Laryngoscope. 1980 Feb;90(2): 217-23.

14. Pyykko I, Selmani Z, Zou J. Low-frequency sound pressure and transtympanic endoscopy of the middle ear in assessment of "spontaneous" perilymphatic fistula. ISRN Otolaryngol. 2012 Aug;2012:137623.

15. Black FO, Lilly DJ, Peterka RJ, Shupert C, HemenwayWG, Pesznecker SC.The dynamic posturographic pressure test for the presumptive diagnosis of perilymph fistulas. Neurol Clin. 1990 May;8(2):361-74.

16. Sugimoto S, Yoshida T, Teranishi M, OkazakiY, Naganawa S, Sone M. The relationship between endolymphatic hydrops in the vestibule and low-frequency air-bone gaps. Laryngoscope. 2018 Jul;128(7): 1658-62.

17. Muchnik C, Hildesheimer M, Rubinstein M, Arenberg IK. Low frequency air-bone gap in Meniere's disease without middle ear pathology: a preliminary report. Am J Otol. 1989 Jan;10(1):1-4.

18. Bohmer A. On the pathomechanism of cochlear dysfunction in experimental perilymph fistulas. Laryngoscope. 1991 Dec;101(12 Pt 1): 1307-12.

19. Simmons FB. The double-membrane break syndrome in sudden hearing loss. Laryngoscope. 1979 Jan;89(1):59-66.

20. Onishi ET, Fukuda Y. Perilymphatic fistula in guinea pigs: natural evolution versus surgical treatment. Braz J Otorhinolaryngol. 2010 Mar-Apr;76(2):178-84.

21. Kohut RI, Hinojosa R, Budetti JA. Perilymphatic fistula: a histopathologic study. Ann Otol Rhinol Laryngol. 1986 Sep-Oct;95(5 Pt 1): 466-71.

22. Black FO, Pesznecker S, Norton T, Fowler L, Lilly DJ, Shupert C, et al. Surgical management of perilymphatic fistulas: a Portland experience.Am J Otol. 1992 May;13(3):254-62.

23. Kohut RI, Hinojosa R, Thompson JN, Ryu JH. Idiopathic perilymphatic fistulas: a temporal bone histopathologic study with clinical, surgical, and histopathologic correlations. Arch Otolaryngol Head Neck Surg. 1995 Apr;121(4):412-20.

24. MeyerhoffWL. Spontaneous perilymphatic fistula: myth or fact. Am J Otol. 1993 Sep;14(5):478-81.

25. Nomura Y, OkunoT, Hara M, Young YH. "Floating" labyrinth: patho- 
physiology and treatment of perilymph fistula. Acta Otolaryngol. 1992;112(2):186-91.

26. Wackym PA, Balaban CD, Zhang P, Siker DA, Hundal JS. ThirdWindow syndrome: surgical management of cochlea-facial nerve dehiscence. Front Neurol. 2019 Dec;10:1281.

27. Park JH, Kim HJ, Kim JS, Koo JW. Costimulation of the horizontal semicircular canal during skull vibrations in superior canal Dehis- cence syndrome. Audiol Neurootol. 2014;19(3):175-83.

28. Deveze A, Matsuda H, Elziere M, IkezonoT. Diagnosis and treatment of perilymphatic fistula.Adv Otorhinolaryngol. 2018;81:133-45.

29. Modugno GC, Magnani G, Brandolini C, Savastio G, Pirodda A. Could vestibular evoked myogenic potentials (VEMPs) also be useful in the diagnosis of perilymphatic fistula? Eur Arch Otorhinolaryngol. 2006 Jun;263(6):552-5. 